\title{
Circuitry Underlying Spectrotemporal Integration in the Auditory Midbrain
}

\author{
Asuman Yavuzoglu, Brett R. Schofield, and Jeffrey J. Wenstrup \\ Department of Anatomy and Neurobiology, Northeast Ohio Medical University, Rootstown, Ohio 44272
}

Combination sensitivity in central auditory neurons is a form of spectrotemporal integration in which excitatory responses to sounds at one frequency are facilitated by sounds within a distinctly different frequency band. Combination-sensitive neurons respond selectively to acoustic elements of sonar echoes or social vocalizations. In mustached bats, this response property originates in high-frequency representations of the inferior colliculus (IC) and depends on low and high frequency-tuned glycinergic inputs. To identify the source of these inputs, we combined glycine immunohistochemistry with retrograde tract tracing. Tracers were deposited at high-frequency $(>56$ $\mathrm{kHz}$ ), combination-sensitive recording sites in IC. Most glycine-immunopositive, retrogradely labeled cells were in ipsilateral ventral and intermediate nuclei of the lateral lemniscus (VNLL and INLL), with some double labeling in ipsilateral lateral and medial superior olivary nuclei (LSO and MSO). Generally, double-labeled cells were in expected high-frequency tonotopic areas, but some VNLL and INLL labeling appeared to be in low-frequency representations. To test whether these nuclei provide low frequency-tuned input to the highfrequency IC, we combined retrograde tracing from IC combination-sensitive sites with anterograde tracing from low frequency-tuned sites in the anteroventral cochlear nucleus (AVCN). Only VNLL and INLL contained retrogradely labeled cells near ( $\leq 50 \mu \mathrm{m})$ anterogradely labeled boutons. These cells likely receive excitatory low-frequency input from AVCN. Results suggest that combination-sensitive facilitation arises through convergence of high-frequency glycinergic inputs from VNLL, INLL, or MSO and low-frequency glycinergic inputs from VNLL or INLL. This work establishes an anatomical basis for spectrotemporal integration in the auditory midbrain and a functional role for monaural nuclei of the lateral lemniscus.

\section{Introduction}

Acoustically guided behavior depends on the integration of information from frequencies across the audible spectrum. Spectral integration is essential for localization of sounds (Hebrank and Wright, 1974, Knudsen and Konishi, 1979; Middlebrooks, 1992; Populin and Yin, 1998), perception of conspecific vocalizations in social interactions (Park and Dooling, 1985; Boothroyd et al., 1996; Shannon et al., 2004; Moore, 2008), and analysis of sonar echoes (Simmons et al., 2004; Genzel and Wiegrebe, 2008). Although classical excitatory tuning curves of peripheral neurons permit some degree of spectral integration, particularly at high sound levels (Sachs and Kiang, 1968; Arthur et al., 1971; Kiang and Moxon, 1974), it is the central auditory system that creates spectrally integrative response properties based on differently tuned inputs. Examples include the broadband inhibition of type IV neurons in the dorsal cochlear nucleus (Young and Brownell,

Received July 11, 2011; revised Aug. 5, 2011; accepted Aug. 12, 2011.

Author contributions: A.Y. and J.J.W. designed research; A.Y. performed research; A.Y., B.R.S., and J.J.W. analyzed data; A.Y., B.R.S., and J.J.W. wrote the paper.

This work was supported by Research Grant R01 DC00937-19S1 and -20 (J.J.W.) and R01 DC04391 (B.R.S.) from the National Institute on Deafness and Other Communication Disorders of the U.S. Public Health Service. We thank Carol Grose for assistance in histological processing and figure preparation, Don Gans (deceased) for software support, Jason Sanchez and Diana Peterson for surgeries, and the Auditory Neuroscience Group at the Northeast Ohio Medical University for discussion of data. We are grateful to the Wildlife Section of the Ministry of Agriculture, Land and Marine Resources of Trinidad for permission to export bats.

Correspondence should be addressed to Dr. Jeffrey Wenstrup, Department of Anatomy and Neurobiology, Northeast Ohio Medical University, 4209 State Route 44, P0 Box 95, Rootstown, 0hio 44272. E-mail: jjw@neomed.edu.

DOI:10.1523/JNEUROSCI.3529-11.2011

Copyright $\odot 2011$ the authors $\quad 0270-6474 / 11 / 3114424-12 \$ 15.00 / 0$
1976; Spirou and Young, 1991), combination-sensitive neurons in bat auditory brainstem and forebrain (Suga et al., 1978; Olsen and Suga, 1991; Mittmann and Wenstrup, 1995; Portfors and Wenstrup, 2001), interaural time difference-selective neurons in the barn owl's auditory midbrain (Takahashi and Konishi, 1986), and harmonic-sensitive neurons in auditory cortex (Kadia and Wang, 2003; Metherate et al., 2005). In each of these, auditory responses show clear evidence of convergent spectral input. These inputs must violate the prevailing tonotopic projection patterns that occur in most nuclei in the ascending pathway. However, the circuitry that underlies this physiological evidence of spectral integration is not well understood.

The current study examines circuitry associated with spectrally integrative responses in the auditory midbrain of the mustached bat, a mammalian species that depends on an analysis of spectrally complex biosonar and social vocalizations. Many neurons in the inferior colliculus (IC), medial geniculate body, and auditory cortex display combination-sensitive facilitation, a form of temporally sensitive spectral integration in which the neuron's excitatory response to its best frequency (BF) is facilitated by signals one to three octaves lower in frequency (Suga et al., 1978; O'Neill and Suga, 1979; Olsen and Suga, 1991; Mittmann and Wenstrup, 1995; Wenstrup, 1999). The combination-sensitive response may be central to the bat's analysis of sonar target distance (O'Neill and Suga, 1979; Suga and O'Neill, 1979; Wenstrup and Portfors, 2011), other features of sonar targets (Suga et al., 1983), and social signals (Ohlemiller et al., 1996; Esser et al., 1997). 

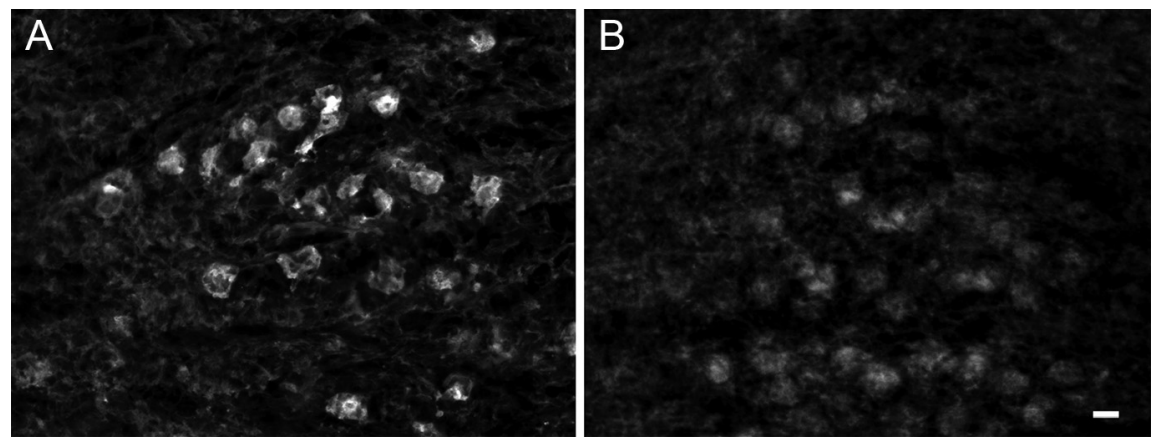

Figure 1. Specific immunolabeling is eliminated in preadsorption control. $A$, Specific anti-glycine labeling of neurons in the medial nucleus of the trapezoid body. $\boldsymbol{B}$, Absence of specific labeling in MNTB from section adjacent to that in $\boldsymbol{A}$. Scale bar (B), $10 \mu \mathrm{m}$.

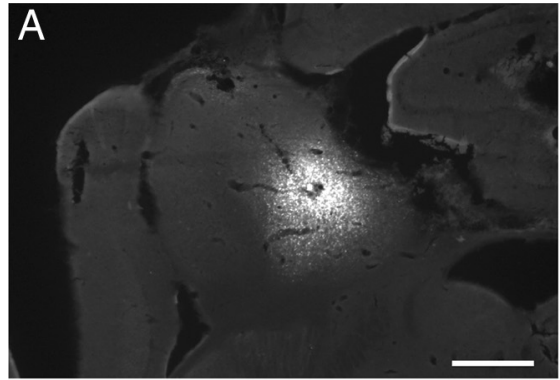

C
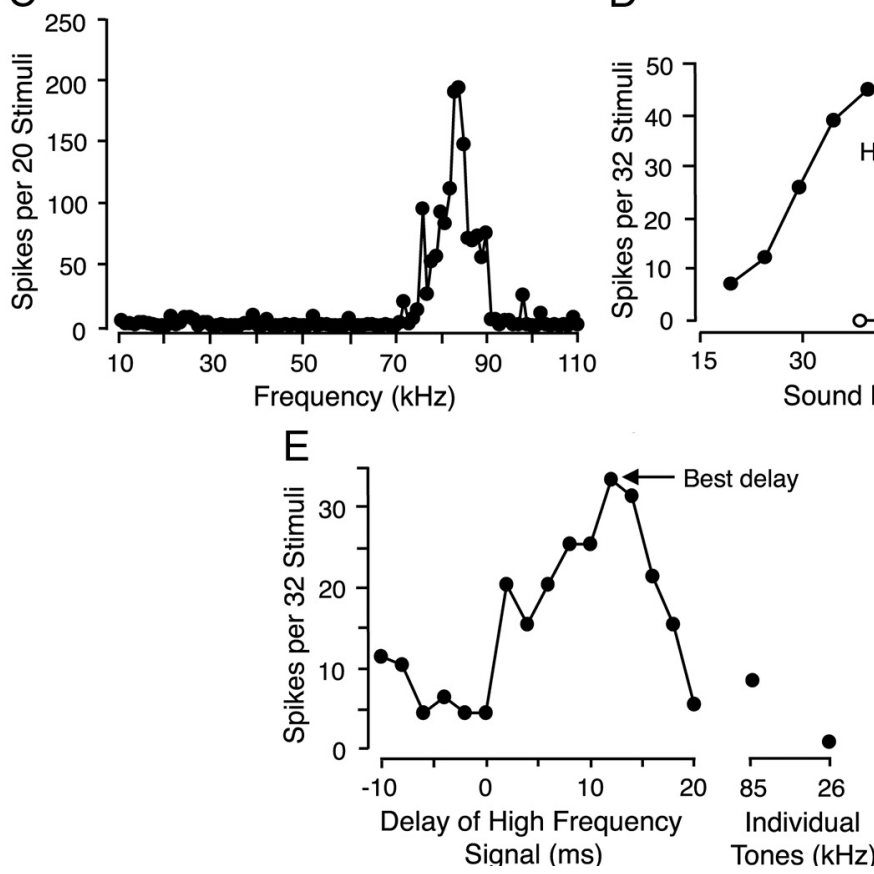

Figure 2. Tracer deposit and response properties at a facilitatory combination-sensitive recording site in the IC. $\boldsymbol{A}$, FG deposit site in left IC. $\boldsymbol{B}$, Reconstruction of penetration culminating in the FG deposit. Black shape indicates center of deposit; gray shape indicates our estimate of the uptake zone for transport. Orientation (D, Dorsal; $M$, medial) and scale apply to both $\boldsymbol{A}$ and $\boldsymbol{B}$. DC, Dorsal cortex of the inferior colliculus; Ex, external nucleus of the inferior colliculus; DPD, dorsoposterior division of the central nucleus of the inferior colliculus, representing frequencies of $\sim 57-64 \mathrm{kHz} ; \mathrm{MD}$, medial division of the central nucleus of the inferior colliculus, representing frequencies $>64 \mathrm{kHz}$. C, Frequency response function obtained with $30 \mathrm{~ms}$ tone bursts attenuated $30 \mathrm{~dB}$ below maximum speaker output. Excitatory responses were observed only in the vicinity of the high BF. $D$, Rate-level functions for BF and best facilitatory low frequency (LF, Low frequency) in response to 4 ms tone bursts. $\boldsymbol{E}$, Combination-sensitive facilitation is evident in two-tone delay tests using 4 ms tone bursts. The response to the BF signal ( $85 \mathrm{kHz}, 29 \mathrm{~dB} \mathrm{SPL}$ ) was strongly facilitated when the best facilitating signal ( $26 \mathrm{kHz}, 64 \mathrm{~dB} \mathrm{SPL})$ was presented $12 \mathrm{~ms}$ in advance of the BF signal. Note also low-frequency inhibition of the response to BF at some delays.
How does facilitatory combination sensitivity arise in the context of the auditory system's tonotopic organization? Facilitatory spectrotemporal interactions underlying combination sensitivity originate in the IC (Portfors and Wenstrup, 2001; Wenstrup and Leroy, 2001, Nataraj and Wenstrup, 2005; Marsh et al., 2006) and depend on distinct sets of glycinergic inputs, one tuned near neurons' high $\mathrm{BF}$ $(>45 \mathrm{kHz})$ and another tuned at least an octave lower in frequency, usually in the range $23-30 \mathrm{kHz}$ (Sanchez et al., 2008). These inputs, when appropriately timed, are thought to activate a postinhibitory rebound excitation that evokes spike discharge (Nataraj and Wenstrup, 2005; Sanchez et al., 2008; Peterson et al., 2008). The present study describes brainstem glycinergic circuitry underlying facilitative combination sensitivity in IC.

\section{Materials and Methods}

Sixteen adult mustached bats (Pteronotus parnellii) of either sex, captured in Trinidad and Tobago, were used to examine sources of inputs to facilitatory combination-sensitive neurons of the IC. The procedures used were approved by the Institutional Animal Care and Use Committee at Northeast Ohio Medical University (Rootstown, $\mathrm{OH}$ ) and follow guidelines set by the National Institutes of Health for the care and use of laboratory animals.

Surgical procedures. Surgery was performed to fix a post on the animal's skull for placement of electrodes in the IC and cochlear nucleus (CN). Before surgery, each animal received an intraperitoneal injection of butorphanol (5 mg/kg, Torbugesic, Fort Dodge Animal Health) and was anesthetized with isoflurane (1.5-2.0\% in oxygen; Abbott Laboratories). After the anesthetic abolished nociceptive reflexes, the hair on the bat's head was removed with depilatory lotion. A midline incision was made in the skin and the underlying muscles were reflected laterally to expose the skull. A metal pin was cemented onto the skull to secure the head to a stereotaxic apparatus used during the experiments, and a tungsten wire was cemented into a small hole in the skull overlying the cerebral cortex to serve as a reference electrode. For access to IC and CN, a small hole was made on the dorsal surface of the skull based on stereotaxic coordinates. A local anesthetic (lidocaine) was applied to the surgical area. The bat recovered from surgery for at least $2 \mathrm{~d}$ before physiological recordings.

Acoustic stimulation and physiological recording. We recently described details of materials and methods for generating acoustic signals and recording neural potentials (Yavuzoglu et al., 2010). Only key elements are included here. Search stimuli were noise bursts of $61 \mathrm{~ms} \mathrm{du}$ ration, $0.5 \mathrm{~ms}$ rise-fall time, 4 per second. Test stimuli included tone bursts or combinations of tone bursts of 4-61 ms, $0.5 \mathrm{~ms}$ rise-fall time, 4 per second. All stimuli were generated digi- 

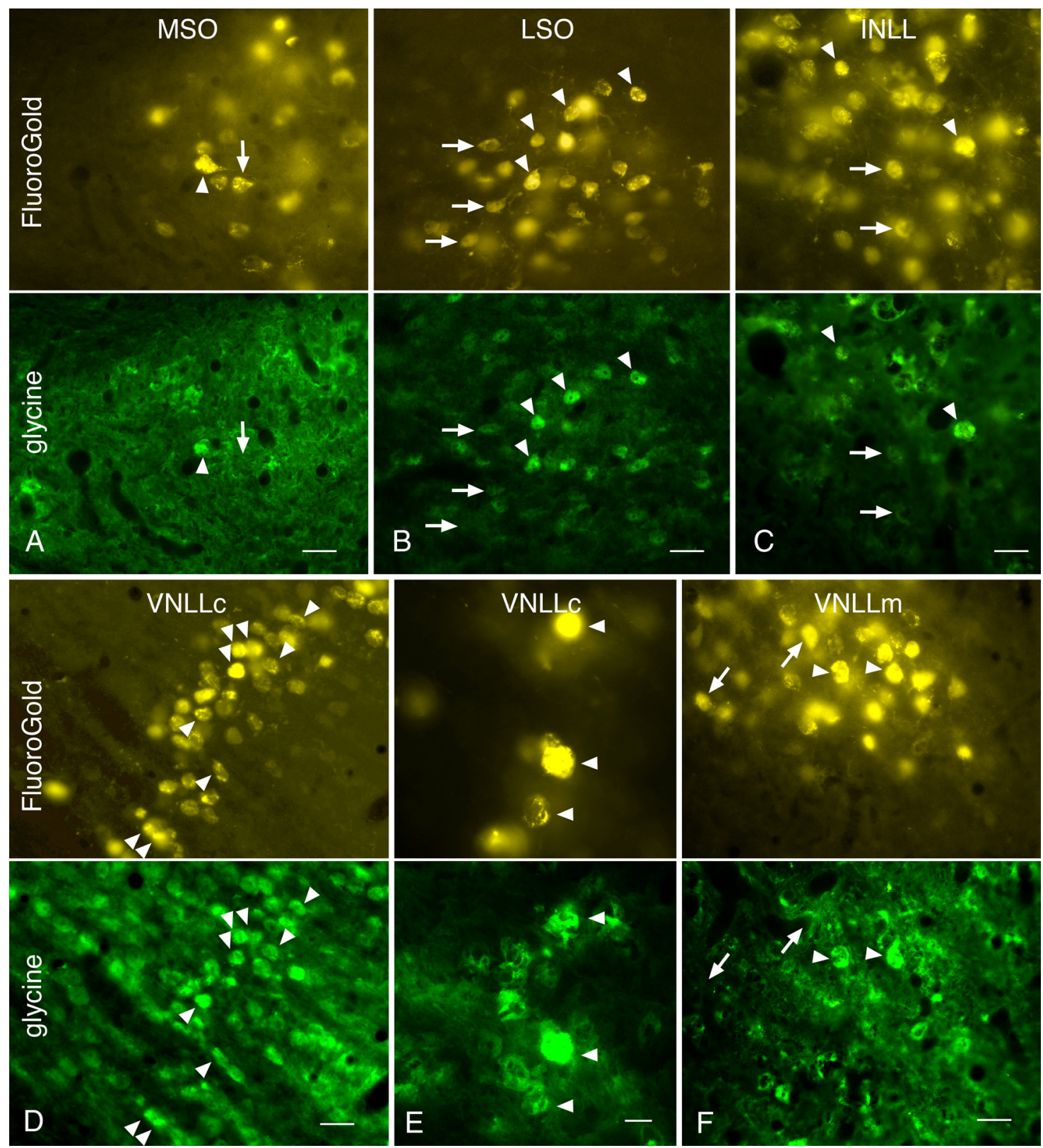

Figure 3. Glycinergic inputs to combination-sensitive sites in IC. Each pair of images displays $\mathrm{FG}$ and Gly + labeling for the same site within brainstem nuclei. Arrowheads indicate cells labeled for both FG and Gly; arrows indicate cells labeled only by FG. A, Cells in the ventral MSO, ipsilateral to FG deposit (case 641). B, Cells in medial LSO, ipsilateral to FG deposit (case 641). C, Cells in ventromedial INLL, ipsilateral to FG deposit (case 641). D, Cells in dorsal VNLLc, ipsilateral to FG deposit (case 670).E, Cells in dorsal VNLLc, ipsilateral to FG deposit (case 641).F, Cells in ventral VNLLm, ipsilateral to FG deposit (case 641). Scale bars: $\boldsymbol{A}-\boldsymbol{E}, 20 \mu \mathrm{m} ; \boldsymbol{F}, 10 \mu \mathrm{m}$.

tally and converted to analog signals at $500 \mathrm{kHz}$. The speaker was placed $10 \mathrm{~cm}$ away from the animal and $25^{\circ}$ into the sound field contralateral to the IC or $25^{\circ}$ into the sound field ipsilateral to the $\mathrm{CN}$ under study. The frequency response of the entire acoustic system had a gradual roll-off of $\sim 3 \mathrm{~dB}$ per $10 \mathrm{kHz}$. Harmonic distortion was not detectable $60 \mathrm{~dB}$ below the signal level.

For electrophysiological recordings, the bat was placed in a heated, custom-made stereotaxic apparatus housed in a single-walled acoustic chamber lined with polyurethane foam to reduce echoes. If the bat showed signs of discomfort, it was lightly sedated with butorphanol $(0.05-0.1 \mathrm{mg} / \mathrm{kg}$, s.c.). Recording sessions typically lasted $4-6 \mathrm{~h}$ and were limited to one session per day.

The evoked activity of clusters of a few neurons (multiunit responses) was recorded using micropipette electrodes filled with $1 \mathrm{M} \mathrm{NaCl}$ (tip diameters: $2-4 \mu \mathrm{m}$ ) or with a neural tracer to mark the recorded neurons (see below, Histological methods). Electrodes were advanced by a hy- draulic micropositioner. For IC experiments, the penetrations were angled $\sim 18^{\circ}$ from lateral to medial to enter the medial, high-frequency subdivision. For $\mathrm{CN}$ experiments, stereotaxic coordinates developed in our laboratory (Marsh et al., 2006) guided penetrations; electrodes were angled $\sim 12^{\circ}$ from medial to lateral from an entry point on the dorsal surface of the cerebellum. Extracellular action potentials from the recording electrodes were amplified, bandpass filtered $(600-6000 \mathrm{~Hz})$, and digitized at a $40 \mathrm{kHz}$ sampling rate. Custom-made software calculated the time of occurrence of the spikes and displayed poststimulus time histograms, rasters, and basic statistics on the neural responses in real time.

Electrodes without tracer were used to map neural activity within the IC or $\mathrm{CN}$. Responses to tone bursts were examined at regular intervals (100-200 $\mu \mathrm{m}$ for IC, $50-100 \mu \mathrm{m}$ for $\mathrm{CN}$ ). We identified best frequency (the frequency requiring the lowest intensity to elicit stimulus-locked spikes) and minimum threshold (MT; the lowest sound level to elicit 
A

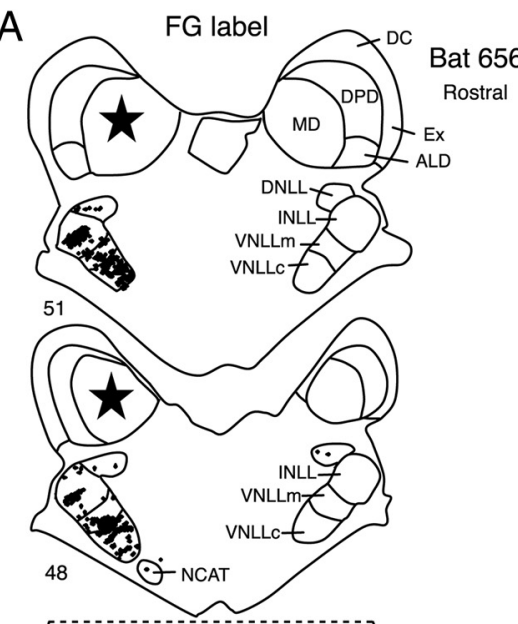

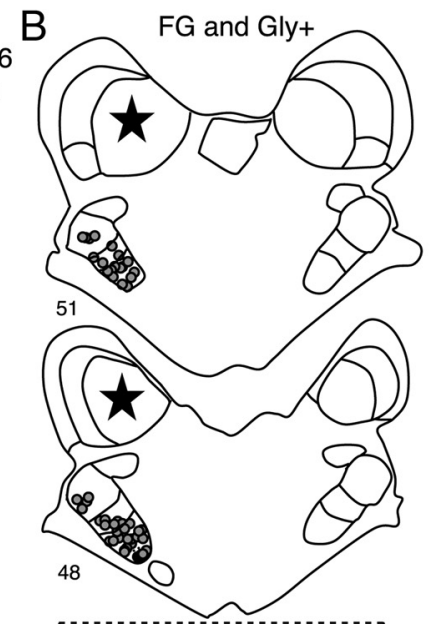
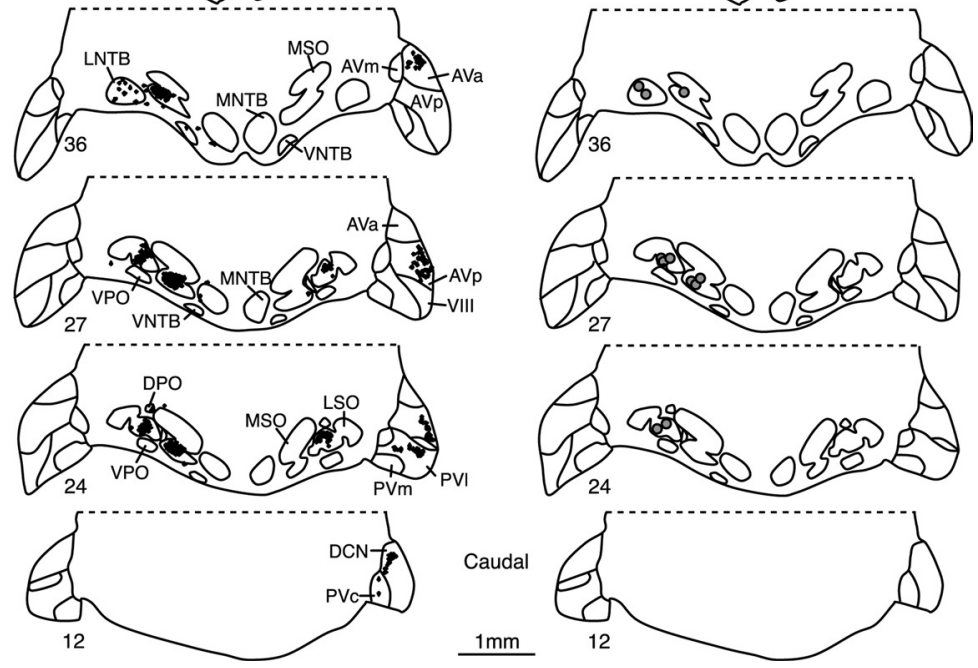

Figure 4. Glycinergic and other inputs to facilitatory combination-sensitive recording sites in IC. A, Distribution of retrogradely labeled cells (FG label) throughout the brainstem after two FG deposits, one illustrated in Figure 1. Transverse sections are arranged from rostral (top) to caudal (bottom). Total number of FG-labeled cells, 1931. ALD, anterolateral division of the central nucleus of the inferior colliculus, representing frequencies below $\sim 57 \mathrm{kHz} ; \mathrm{AVa}$, anterior division of anteroventral cochlear nucleus; $\mathrm{AVm}$, marginal division of anteroventral cochlear nucleus; $A V p$, posterior division of anteroventral cochlear nucleus; $D C$, Dorsal cortex of the inferior colliculus; DCN, dorsal cochlear nucleus; DPD, dorsoposterior division of the central nucleus of the inferior colliculus, representing frequencies of $\sim 57-64 \mathrm{kHz}$; DP0, dorsal periolivary region; $\mathrm{Ex}$, external nucleus of the inferior colliculus; LNTB, lateral nucleus of trapezoid body; MD, medial division of the central nucleus of the inferior colliculus, representing frequencies $>64 \mathrm{kHz}$; NCAT, nucleus of the central acoustic tract; PVc, caudal division of posteroventral cochlear nucleus; PVI, lateral division of posteroventral cochlear nucleus; PVm, medial division of posteroventral cochlear nucleus; VIII, root of eighth cranial nerve; VNTB, ventral nucleus of trapezoid body; VPO, ventral periolivary nucleus. $B$, Distribution of double-labeled cells (FG and GLY+) throughout the auditory brainstem. Most double-labeled cells are in the lateral lemniscal nuclei, particularly in VNLLc. Total number of double-labeled cells, 184; double-labeled cells in VNLLc, 98 (53\%). Tracer deposit sites are depicted here by a star. presented $10 \mathrm{~dB}$ above its threshold. The second tone (a lower frequency signal) was set to frequencies within the fundamental of the biosonar call $(23-30 \mathrm{kHz})$ and varied over a range of intensities and timing (delays) relative to the $\mathrm{BF}$ signal. Delay sensitivity was evaluated in $2 \mathrm{~ms}$ steps. Typically, short duration stimuli (4 ms) were used to reveal maximum temporal sensitivity. Neurons were considered to show low frequency (combination-sensitive) facilitation of high-BF signals if the response to the combination of tones was at least $20 \%$ greater than the sum of the individual responses to the low and highfrequency tones. These criteria have been used extensively in our past studies (e.g., Portfors and Wenstrup, 1999; Sanchez et al., 2008; Gans et al., 2009). After completion of all tests, a tracer was deposited iontophoretically from the recording electrode.

In the mustached bat IC, at least $25 \%$ of high-BF neurons display combination-sensitive facilitation (Portfors and Wenstrup, 1999, 2001; Nataraj and Wenstrup, 2005) that is distributed throughout the dorsoventral and rostrocaudal dimensions of the central nucleus of the IC (Portfors and Wenstrup, 2001). As a result, any tracer deposit in these high-BF representations will identify inputs to large numbers of facilitatory neurons. To maximize retrograde labeling of the neurons providing facilitatory input, we often made two deposits of FluoroGold (FG, 2\% in filtered $0.9 \%$ saline; FluoroChrome) at different physiologically identified sites. FluoroGold was ejected using pulsed current ( $+5 \mu \mathrm{A} ; 7 \mathrm{~s}$ on/off) for 5-8 min. Due to the smaller size of $\mathrm{CN}$, we made single deposits of anterograde tracers that featured more focused deposit sites. FluoroRuby (FR, tetramethylrhodamine dextran, molecular weight $10,000,8-10 \%$ in filtered $0.9 \%$ saline; Invitrogen) was ejected using pulsed current $(+5$ $\mu \mathrm{A} ; 7 \mathrm{~s}$ on/off) for 10-20 min. Biotinylated dextran amine (10\% in filtered $0.9 \%$ saline; Invitrogen) was ejected using pulsed current $(+5 \mu \mathrm{A} ; 7 \mathrm{~s}$ on/off) for 10-15 min. For all deposits, the tracer pipette was kept in the brain for at least $5 \mathrm{~min}$ after the deposit to minimize accidental ejection of residual tracer during the retraction of the electrode. In some animals, both IC and the contralateral $\mathrm{CN}$ received tracer deposits. Typically, IC deposits at high-BF, combination-sensitive sites were placed first, followed by deposits at low-BF sites in CN.

Histological methods. Animals used in transstimulus-locked spikes). In IC, we evaluated combination sensitivity as described below. These penetrations established the borders and frequency organization in the area of interest as well as the locations of response properties for subsequent tracer deposits.

Tracer deposits. Tracer deposits in IC were made at high-BF sites $(\geq 57$ $\mathrm{kHz}$ ) showing combination-sensitive facilitation, while deposits in $\mathrm{CN}$ were made at either low-BF sites $(23-30 \mathrm{kHz})$ or at high-BF sites $(\geq 57$ $\mathrm{kHz}$ ). After an electrode without tracer revealed the presence of response properties appropriate for a tracer deposit, the electrode was replaced with a tracer-filled electrode at the same location. With the tracer-filled electrode, multiunit response properties at the site to receive the deposit were examined in detail. First, BF and MT were obtained audiovisually. Subsequent quantitative tests examined responsiveness across a broad frequency range $(10-110 \mathrm{kHz})$ at one or more attenuation values and across a range of sound levels at BF. We used a two-tone stimulus paradigm to evaluate the presence of low frequency-evoked interactions with high-BF excitatory responses. One tone was set to the multiunit BF and port studies survived 5-8 d before they were euthanized. Each animal was killed with an overdose of Fatal Plus ( $>100 \mathrm{mg} / \mathrm{kg}$, i.p., Vortech). Following loss of corneal and withdrawal reflexes, the animal was perfused through the left ventricle with phosphate-buffered saline, followed by $4 \%$ paraformaldehyde in $0.1 \mathrm{~m}$ phosphate buffer, $\mathrm{pH}$ 7.4. The skull was removed and the brain was blocked in a transverse plane angled $15^{\circ}$ dorsocaudal to ventrorostral. After removal, the brain was stored overnight at $4^{\circ} \mathrm{C}$ in a $30 \%$ sucrose-phosphate buffer solution and then sectioned on a freezing microtome at a thickness of $35-40 \mu \mathrm{m}$. Sections were collected from the cochlear nucleus through the auditory cortex in three alternating series. Typically, one series was mounted on gelatincoated slides and stained with cresyl violet to identify cytoarchitectural boundaries. One or two series to be used for fluorescence were mounted on gelatin-coated slides and coverslipped with DPX.

In several experiments, the third series was reserved for glycine immunocytochemistry using antisera compatible with paraformaldehyde fixa- 
tion. Preparation and evaluation of polyclonal rabbit anti-glycine by the manufacturer (Immunosolution Pty, catalog no. IG1001) followed established procedures (Pow and Crook, 1993; Pow et al., 1995). Procedures for use in mustached bats and labeling in the auditory brainstem were described previously (Yavuzoglu et al., 2010). Briefly, sections were placed in a blocking solution and then incubated with polyclonal rabbit anti-glycine (dilution 1:1000) followed by Alexa Fluor 488-goat antirabbit secondary antibody (1:250; Invitrogen, \#A11008). Sections were mounted, cleared, and coverslipped with DPX. Optimum labeling was determined by testing antibody dilutions of 1:750, 1:1000, and 1:1500.

Several controls were conducted to demonstrate that anti-glycine staining was specific. In one bat, a preadsorption control was performed with the manufacturer's thyroglobulin-glycine antigen (Immunosolution, catalog no. GLYG02). Antigen ( $25 \mu \mathrm{l}$ per $\mathrm{ml}$ of blocking solution) was incubated overnight at $4^{\circ} \mathrm{C}$ with the primary antibody (1:1000 dilution). We observed specific labeling of cells in the medial nucleus of the trapezoid body (MNTB) in normal anti-glycine immunocytochemistry (Fig. 1, A) but no specific labeling of MNTB cells in the preadsorbed control (Fig. 1, $B)$. The nonspecific labeling of MNTB cells in the preadsorbed control was comparable to cell staining in the IC, which does not contain glycinergic cells (Winer et al., 1995). We also performed immunocytochemistry with omission of primary or secondary antibodies. Specific staining was eliminated in these omission control series.

Data analysis. Retrogradely labeled and immunolabeled cells and anterogradely labeled presumptive boutons in the brainstem were plotted using a Zeiss Axioplan II microscope and Neurolucida reconstruction system (MBF Bioscience). We used several criteria to identify labeled cells and axons. Labeling must be clearly differentiated from the background labeling in the nucleus and surrounding region.

This is accomplished by examining each area in the "appropriate" filter as well as other filters; the latter comparison reveals nonspecific crossover that is characteristic of background labeling or especially intense "real" labeling. By comparing across filters and areas, it is possible to attain a consistent decision on labeling. To further ensure objective analyses, each conclusion that depended on the presence or absence of double-labeling (FG and anti-glycine) or proximity of labeling (FGlabeled cells near FR-labeled axons) was confirmed by direct observation in the microscope by each of the authors. Outlines of brainstem nuclei and subdivisions were drawn in Neurolucida from the unstained DPX-mounted sections, with adjacent Nissl-stained sections serving as references. Cytoarchitectonic boundariesare based on previous work in mustached bats (Zook and Casseday, 1982; Zook et al., 1985; Kössl and Vater, 1990; Vater, 1995).

To compare the retrograde labeling in different brainstem nuclei, for each case we counted the FG-labeled cells in each nucleus in one series of sections. We then divided that number by the total number of FG-labeled cells to represent the percentage of total FG-labeled cells in each nucleus. We then computed the mean and sample standard deviation of those percentages for each nucleus across the nine successful transport cases; this is expressed as "Mean Percentage of FG-Labeled Cells." In a similar way, we computed the "Mean Percentage of Double-Labeled Cells"; for each case, we counted the double-labeled cells and calculated the per- centage of the population that was present in each nucleus. We then computed the mean and standard deviation of these percentages across the five successful double-labeling cases.

To estimate the potential for synaptic contacts between $\mathrm{CN}$ axonal terminals and brainstem cells that project to combination-sensitive recording sites in IC, we used the Neurolucida plots to identify retrogradely labeled cells that were within $50 \mu \mathrm{m}$ of an anterogradely labeled terminal. These cells are considered to be "proximity labeled." In intermediate nucleus (INLL) and ventral nucleus of the lateral lemniscus (VNLL), where proximity-labeling occurs, dendrites extend at least $50 \mu \mathrm{m}$ from the soma (Covey and Casseday, 1991; Vater et al., 1997). We computed "Mean Percentage of Proximity-Labeled Cells" (see Fig. 12) as described above; for each case, we counted proximity-labeled cells and calculated the percentage of that population that was present in each nucleus. We then computed the mean and standard deviation of these percentages across the five successful proximity labeling cases.

Tracer deposit sites and brainstem labeling were photographed with either a Zeiss Axiocam HRc or HRm camera and AxioVision software (version 4.6; Zeiss) mounted on a Zeiss Imager Z1m fluorescence microscope or a SPOT RT3 camera and SPOT Advanced Plus imaging software (version 4.7) mounted on a Zeiss Axio Imager M2 fluorescence microscope. Adobe Photoshop CS3 was used to overlay injection images, adjust brightness and contrast, and add labels. 
A Bat 656

$$
\text { - FG cells }
$$
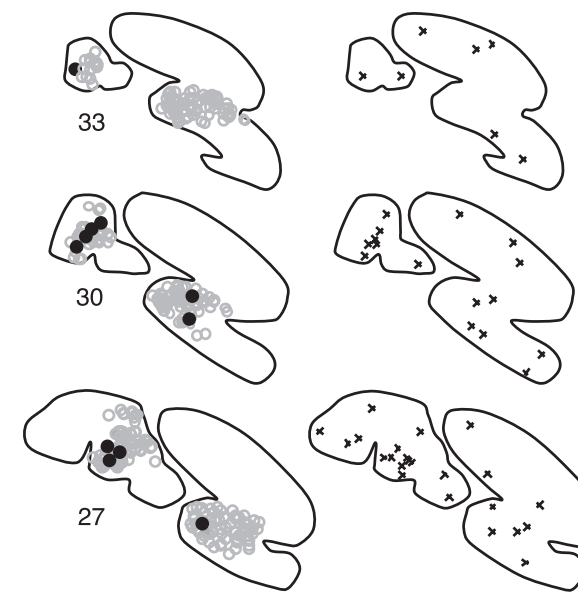

* Gly+ cells
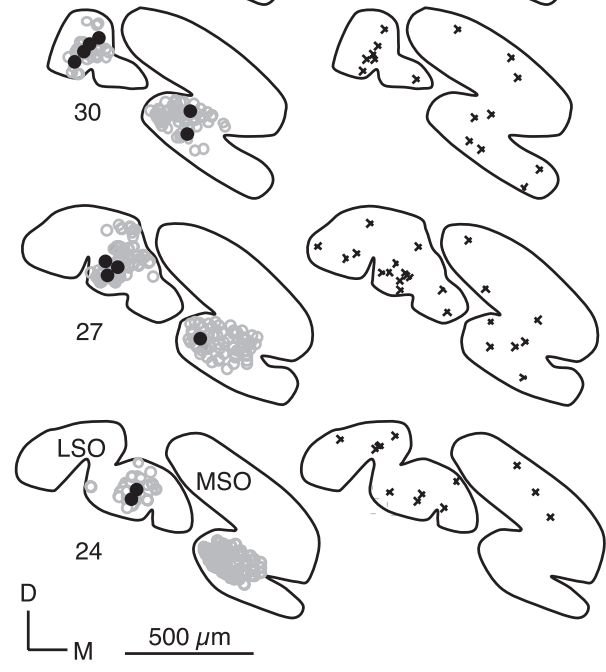

\section{B Bat 641}

- FG cells

Rostral

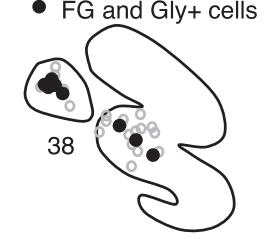

$\times$ Gly + cells
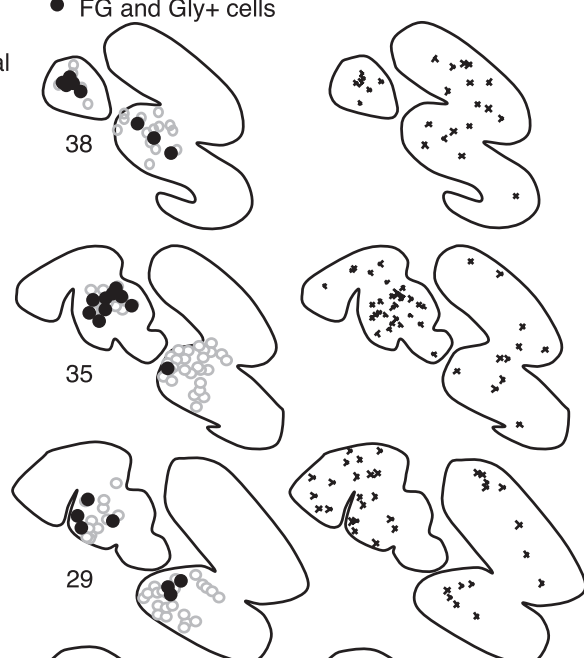

Caudal

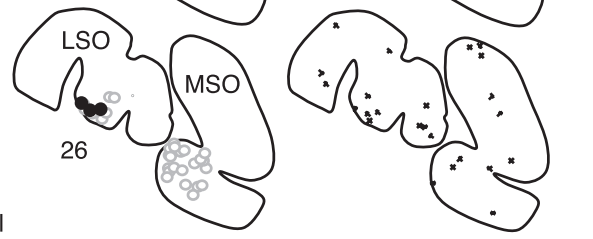

Figure 6. Retrogradely labeled (gray circles) and double-labeled (black circles) cells were present only in high-frequency regions of the ipsilateral LSO and MSO after combination-sensitive FG deposits in IC. Results in $\boldsymbol{A}$ and $\boldsymbol{B}$ are from two separate cases. Retrograde ( $\mathrm{FG}$ ) and double labeling ( $\mathrm{FG}$ and $\mathrm{Gly}+$ ) is shown in left columns of $\boldsymbol{A}$ and $\boldsymbol{B}$, while glycine labeling is shown in right column (black symbols). In all such cases, there was a single focus of labeled cells in high-frequency regions of LSO and MSO. D, Dorsal; M, medial.

\section{Results}

\section{Glycinergic inputs to combination-sensitive recording sites in IC}

In nine cases with successful retrograde transport, deposits of FG were placed at caudal IC recording sites with best excitatory frequencies in the range $58-84 \mathrm{kHz}$. The neurons exhibited little or no excitatory response to sounds between 23 and $30 \mathrm{kHz}$, but an appropriately timed signal within that range evoked an enhanced response to the high-BF sound (combination-sensitive facilitation). Figure 2 illustrates response properties at one of these deposit sites (Fig. $2 \mathrm{~A}$ ) that was made along an electrode penetration that encountered tonotopically increasing BFs from 60 to $93 \mathrm{kHz}$ (Fig. $2 \mathrm{~B}$ ). A facilitated combination-sensitive response was identified at the location of an $85 \mathrm{kHz}$ BF response (Fig. 2C). The multiunit recording showed a weakly nonmonotonic response to $\mathrm{BF}$ signals and virtually no response to a low-frequency (LF) signal at $26 \mathrm{kHz}$ (Fig. 2D). When the two signals were combined, the response to the BF sound was inhibited at some delays and facilitated at other delays (Fig. 2E). For this recording site, the strongest facilitation occurred at a BF delay of $12 \mathrm{~ms}$. The connections underlying the strong facilitation of combinationsensitive IC neurons are the focus of the current study.

FG deposits at facilitatory combination-sensitive sites in IC from the nine cases resulted in many well labeled somata throughout the brainstem (Figs. 3, 4A). In five of these cases, we also examined glycine immunoreactivity in the structures that contained FG-labeled cells (Figs. 3, 4B). Retrograde labeling from a case with two FG deposits having BFs of 79 and $85 \mathrm{kHz}$ (Fig. 2) is illustrated in representative sections throughout the brainstem (Fig. $4 A$ ), while a quantitative summary of all nine cases is shown in Figure $5 A$. Roughly half of all labeled cells were located in the nuclei of the lateral lemniscus, mainly in the ipsilateral VNLL and INLL. Other major inputs included the ipsilateral medial superior olive (MSO) and the contralateral CN. In general, the locations of FG-labeled cells within auditory brainstem nuclei were restricted in accord with the tonotopically restricted locations of the IC deposit sites (Fig. 4A). These retrograde labeling patterns after restricted IC deposits are consistent with previous studies in the mustached bat (Frisina et al., 1989; Ross et al., 1988; Wenstrup et al., 1994, 1999).

Anti-glycine immunolabeling (Gly+) was present in nearly every major auditory brainstem structure. Noteworthy exceptions were the IC and dorsal nucleus of the lateral lemniscus (DNLL), which had no labeled cell bodies. These patterns of labeling correspond closely to previous reports in mustached bats (Vater, 1995; Winer et al., 1995; Kemmer and Vater, 1997; Yavuzoglu et al., 2010). Many nuclei contained both glycineimmunolabeled cells and retrogradely labeled cells; we focus here on those nuclei that contained double-labeled cells.

Double-labeled cells were readily identified in numerous auditory brainstem nuclei (Fig. 3). The majority-over twothirds-of all double-labeled cells was in the VNLL (Fig. 3D-F, $4 B, 5 B)$. The columnar part of the VNLL (VNLLc) had the highest number of double-labeled cells among auditory brainstem structures ( $49 \%$ of all double-labeled cells), while the multipolar part of the VNLL (VNLLm) (Fig. $3 F$ ) and the intermediate nucleus of the lateral lemniscus (INLL) (Fig. 3C) had significant numbers, 19 and 11\%, respectively (Fig. 5B). Among structures of the superior olivary complex, the ipsilateral MSO (Fig. $3 A$ ) and the lateral superior olive (LSO) (Fig. 3B) had the largest numbers of double-labeled cells, $6 \%$ and $8 \%$ respectively (Fig. $5 B$ ). Other brainstem structures had few $(<2 \%)$ or no double-labeled cells. 
A Bat 656

- FG cells
- FG and Gly+ cells
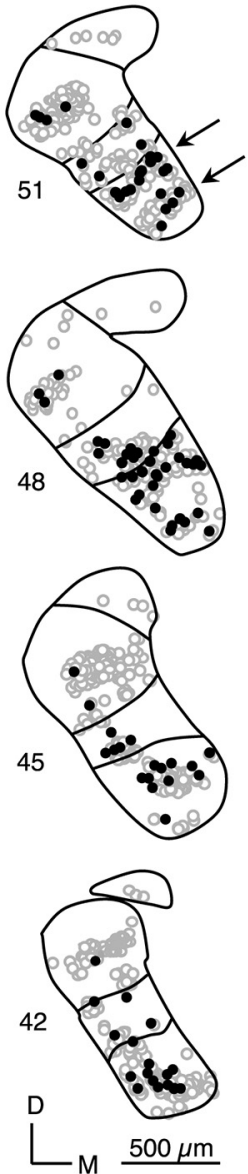

× Gly+ cells
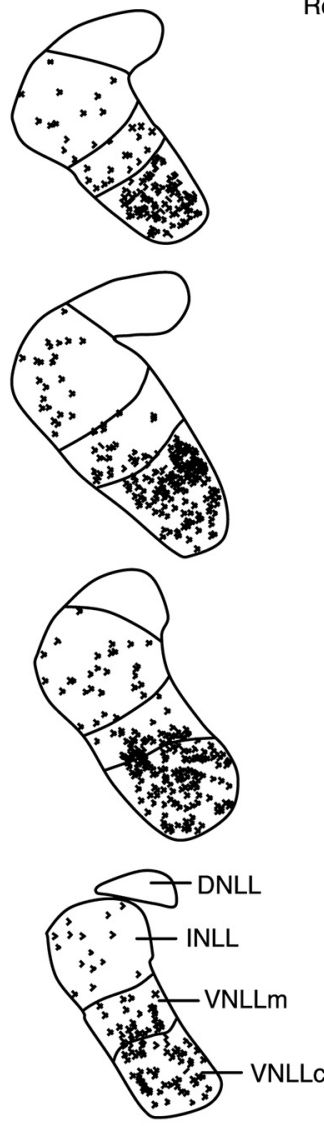

Caudal

Rostral
B Bat 641

- FG cells

- FG and Gly+ cells

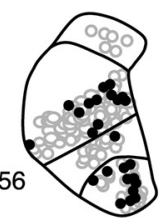

$\times$ Gly+ cells
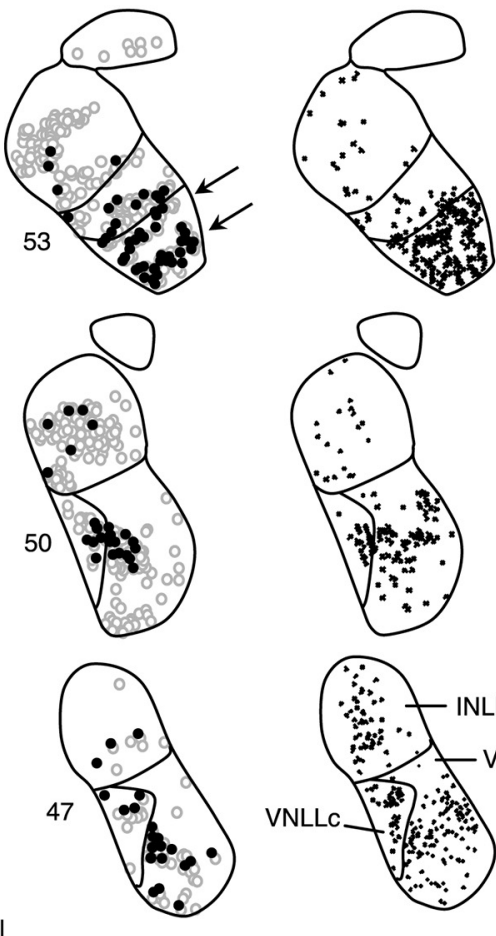

Figure 7. Retrogradely labeled (gray circles) and double-labeled (black circles) cells were present at multiple foci in NLL after combination-sensitive FG deposits in IC. $\boldsymbol{A}, \boldsymbol{B}$, Results from same two cases as illustrated in Figure 5. Arrows indicate bands of label in VNLLc. Protocol same as for Figure 5. D, Dorsal; M, medial.

The results suggest that the ipsilateral VNLL, INLL, MSO, and LSO supply nearly all glycinergic input to facilitative combinationsensitive neurons of IC.

Due to the presence of significant numbers of double-labeled cells in superior olivary and lateral lemniscal nuclei, we compared the locations of the labeled cells within these nuclei to their well documented tonotopic organizations (Covey et al., 1991; Grothe et al., 1992; Vater, 1995). The dorsolateral parts of these nuclei represent the lowest frequency sounds in the bat's audible range, while higher frequency sounds are represented more ventromedially. Both FG and double-labeled cells were consistently located midway or more along the low- to high-frequency axes of MSO and LSO (Fig. 6). No FG-labeled cells were present in the dorsolateral (low frequency) MSO or LSO. These findings indicate that ipsilateral LSO and MSO are likely sources of high-frequency but not low-frequency inputs to facilitated combination-sensitive neurons of IC.

Among lateral lemniscal nuclei, double-labeled cells were present in INLL and VNLL (Fig. 7). In INLL, most double-labeled cells were placed laterally and ventrally, consistent with high-BF tonotopic representations (Ross et al., 1988; Wenstrup et al., 1994, 1999; Yavuzoglu et al., 2010). However, some double-labeled cells were located in the medial INLL (e.g., Fig. 7B, section 56), near locations that contain low-frequency tuned neurons (Yavu- zoglu et al., 2010). In VNLL, double-labeling patterns were more complex than those in INLL, occurring in multiple foci within both VNLLm and VNLLc. For example, both experiments in Figure 7 reveal two mediolateral bands of labeling in VNLLc, one located dorsally and another placed more ventrally (arrows in Fig. 7A, section 51, and Fig. $7 B$, section 53). The dorsal band in VNLLc is associated with labeling after deposits of retrograde tracer deposits in low-frequency IC representations (Ross et al., 1988; Wenstrup et al., 1994; 1999). These results suggest that both low-frequency and high-frequency tuned neurons in VNLLc and VNLLm project to facilitated combination-sensitive sites in IC. To explore this possibility, we performed the combined tracing experiments described in the next section.

\section{$\mathrm{CN}$ inputs to neurons projecting to combination-sensitive IC neurons}

In five cases, we sought to identify the locations of brainstem auditory neurons that receive low-frequency input and that project to combination-sensitive recording sites in IC. We combined anterograde tracing after FR deposits in the low-frequency AVCN (e.g., Fig. $8 A, B$ ) with retrograde tracing after FG deposits at combination-sensitive IC recording sites. Although we sometimes observed apparent contacts between FR-labeled boutons and FG-labeled cells, we more often observed FR-labeled boutons 
A

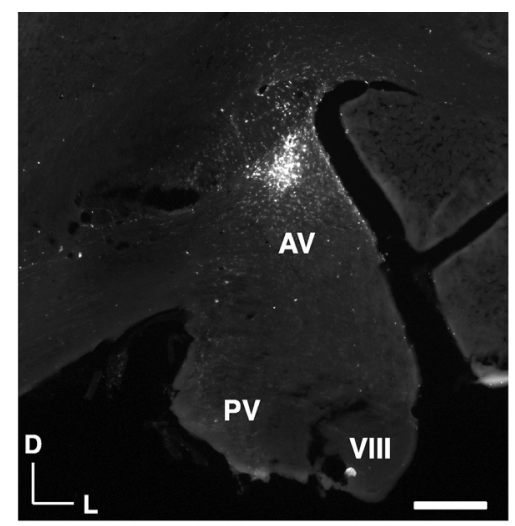

B

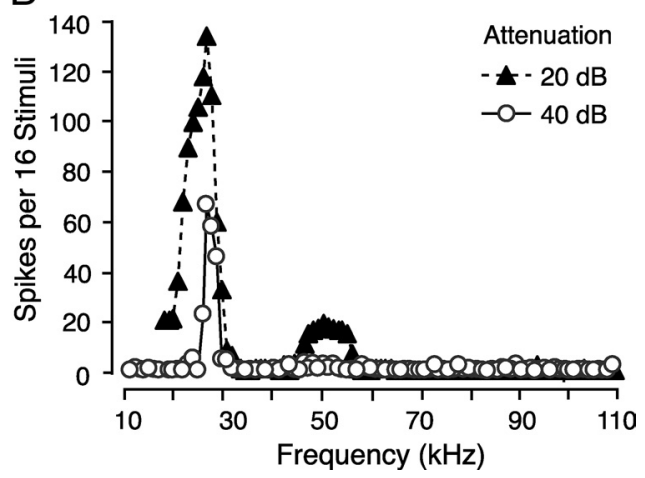

Figure 8. Features of auditory responses associated with deposits of anterograde tracer in AVCN. A, FR deposit at recording site tuned to $26 \mathrm{kHz}$. Scale bar, $500 \mu \mathrm{m}$. AV, Anteroventral cochlear nucleus; PV, posteroventral cochlear nucleus; VIII, root of eighth cranial nerve. $\boldsymbol{B}$, Acoustic frequency response obtained at deposit site is shown in $\boldsymbol{A}$ for two attenuation levels. Sound duration was $30 \mathrm{~ms}$. Note that high-frequency tones in the range of the BFs of combination-sensitive neurons studied here $(58-84 \mathrm{kHz})$ did not evoke spikes, even at high sound levels. Tonotopic progression in the penetration that included this recording site is shown in Figure 10, section 38 .

in the vicinity of FG-labeled cells (Fig. 9C-E), which we term "proximity labeling." To characterize the overlap between FR and FG labeling patterns, we plotted proximity-labeled cells, quantified as those FG-labeled cells within $50 \mu \mathrm{m}$ of FRlabeled boutons.

The first case includes the $\mathrm{CN}$ deposit and physiology (BF, 26 $\mathrm{kHz}$ ) illustrated in Figures 8 and 10. The IC deposits in this case were associated with high-frequency excitation (BFs of 60 and 75 $\mathrm{kHz}$ ) and delay-tuned facilitation by frequencies in the 25-28 $\mathrm{kHz}$ range (Fig. $10 \mathrm{~B}$ ). Plots of FG-labeled cells and FR-labeled boutons (Fig. 10A) revealed overlap in VNLLc, Proximity labeling was present in VNLLm and INLL, but was most prominent in VNLLc (70\%). No proximity labeling was observed in MSO or LSO. Results were similar in a second case (Fig. 11) in which IC deposit sites had BFs of 74 and $85 \mathrm{kHz}$ with delay-tuned facilitation by $25-27 \mathrm{kHz}$, and the $\mathrm{CN}$ deposit had a BF of $23 \mathrm{kHz}$. In this case, proximity-labeled cells were observed only in VNLLc (58\%) and VNLLm (42\%). In both cases, the restricted AVCN deposit sites were evident in anterograde labeling patterns in the superior olive and IC that corresponded to low-frequency tonotopic locations.

Across the five cases that had both IC and CN injections, proximity-labeled cells were most common in VNLLc, where on average $60 \%$ of the proximity-labeled cells were observed. Proximity labeling also occurred in VNLLm and INLL in lesser quantities but did not occur in any other brainstem nucleus (Fig. 12).
These labeling patterns suggest that some neurons within VNLL and INLL receive input from low-frequency tuned neurons in AVCN and project to IC regions containing combinationsensitive neurons.

\section{Discussion}

Combination-sensitive facilitation is characterized by an enhanced response when two sounds of different frequencies occur in a specific temporal relationship (Suga, 1989). The frequency difference can be substantial, up to three octaves, and the temporal relationship can vary from zero to tens of milliseconds. These characteristics suggest that combination sensitivity could contribute to the analysis of complex sounds.

Echolocating animals have provided a focus of research on combination sensitivity. Echolocation depends on analysis of the precise temporal relationships between sonar echo components that can be quite different in frequency. Combination sensitivity is also likely to play a substantial role in analysis of social vocalizations, which typically contain many frequencies in a complex temporal pattern. Segments or syllables of vocalizations typically comprise multiple frequency components in a stereotypical temporal pattern. Combination-sensitive cells could be particularly efficient for detecting and distinguishing syllables or syllable combinations. Accordingly, combination sensitivity has been observed in many nonecholocating animals, including frogs (Fuzessery and Feng, 1983), songbirds (Margoliash and Fortune, 1992), mice (Portfors and Felix, 2005), and primates (Kadia and Wang, 2003). The characteristics of combination sensitivity are similar across species, suggesting that similar mechanisms may be operating. Our work supports the idea that combination sensitivity is a widespread, generalized phenomenon and, furthermore, closely ties this type of analysis to lateral lemniscal nuclei. In fact, the present data provide new insights into specific functions served by the ventral and intermediate lateral lemniscal nuclei.

\section{Auditory brainstem circuitry underlying combination-sensitive facilitation}

Although facilitated combination-sensitive neurons in IC receive glutamatergic and GABAergic inputs, these are unrelated to the facilitatory interaction (Sanchez et al., 2008). Instead, blockade of glycinergic inputs to IC cells eliminates facilitative combination sensitivity (Wenstrup and Leroy, 2001; Nataraj and Wenstrup, 2005; Sanchez et al., 2008). Given the apparent lack of such sensitivity in lower auditory nuclei (Portfors and Wenstrup, 2001; Marsh et al., 2006), we concluded that the facilitation is created by convergence of two sets of glycinergic inputs onto IC neurons. One input is driven by high frequencies, matching the tonotopic location of the target cell and the BF of its glutamatergic inputs. A second input, also glycinergic, is tuned to frequencies 1-3 octaves below BF. Neither glycinergic input evokes spikes on its own, but the combination of both inputs, with appropriate timing, activates spike discharge. How does this occur? Since glycine is generally considered inhibitory, we believe that each input activates an inhibitory conductance related to stimulus onset that, in turn, leads to rebound excitation. If the two rebounds coincide, the neuron discharges a spike. The time window for this coincidence detection mechanism is about $5 \mathrm{~ms}$ on average and probably depends on the duration of the rebound (Gans et al., 2009).

The present goal was to identify candidates for the facilitating low-frequency and high-frequency glycinergic inputs. These inputs must originate in lower auditory nuclei, the only auditory structures that contain glycinergic neurons (Winer et al., 1995). 
The results identify four likely candidates, VNLL, INLL, LSO, and MSO (Fig. 12), which together account for $>93 \%$ of double-labeled (Gly+ retrograde) neurons projecting to IC combination-sensitive sites.

\section{Sources of facilitating}

high-frequency input

VNLL, INLL, LSO, and MSO contained double-labeled (Gly+ retrograde) neurons in locations consistent with highfrequency responses. Cells in the ipsilateral LSO, primarily excited by the ipsilateral ear (Covey et al., 1991), are unlikely to be excited by the contralateral stimuli used here. Thus, they are unlikely to contribute significantly to combination-sensitive responses that we observed. MSO neurons in mustached bats have appropriate physiological properties, e.g., onset responses and contralateral excitation (Covey et al., 1991), but only a small number provide glycinergic input to IC combination-sensitive neurons. In contrast, glycinergic cells are prominent in the VNLL and, less so, in the INLL (Winer et al., 1995; Vater et al., 1997). Moreover, many VNLL neurons and some INLL neurons have onset responses corresponding to those expected of inputs contributing to facilitation (Portfors and Wenstrup, 2001). We conclude that VNLL and perhaps INLL provide most high-frequency glycinergic input that underlies combination-sensitive facilitation. These nuclei share features across many mammals, so they could also underlie facilitative interactions observed in other mammalian species.

\section{Sources of facilitating low-frequency input}

The low-frequency input is also likely to originate from VNLL and INLL (Fig. 12). In VNLLc, a region known in bats to be almost exclusively glycinergic (Winer et al., 1995; Vater et al., 1997), low frequencies are located dorsally or medially, where we observed retrograde labeling after tracer deposits at IC combination-sensitive recording sites (Ross et al., 1988; Wenstrup et al., 1994, 1999). VNLLm has fewer glycinergic neurons, but many are labeled after IC combination-sensitive deposits. In INLL, some double labeling occurred in the medial part that represents frequencies in the $20-30 \mathrm{kHz}$ range (Ross et al., 1988; Wenstrup et al., 1994, 1999; Yavuzoglu et al., 2010). Our anterograde labeling demonstrates that low-frequency AVCN axons terminate close to or on nuclear lateral lemniscus (NLL) neurons that project to combination-sensitive sites in IC. MSO and LSO neurons that project to these IC sites are in high-frequency areas and do not receive low-frequency AVCN input. We conclude that VNLL and INLL are likely sources of the low-frequency, glycinergic inputs that underlie combination-sensitive facilitation.

\section{Technical limitations}

Our tracers are unlikely to have labeled all cells or axons that are involved in the circuits of interest. It is also unlikely that our immunohistochemistry labeled every glycinergic cell (Yavuzoglu et al., 2010). More importantly, these experiments cannot demonstrate functional circuitry at a cell-to-cell level, so it remains for more detailed studies to demonstrate functional synapses of ventral CN neurons onto NLL neurons, which in turn provide glycinergic inputs onto combination-sensitive IC neurons. Nonetheless, these results identify likely candidates for the key elements required in the circuitry underlying combinationsensitive facilitation: the presence of glycinergic inputs to the appropriate regions of IC and the strong likelihood that these include both low and high frequency-tuned auditory brainstem neurons with appropriate temporal response properties. When combined with previous anatomical studies of auditory brainstem pathways in the mustached bat and physiological studies of combination-sensitive facilitation in IC, this work provides some of the strongest evidence for a circuit underlying spectral integration in the auditory system.

\section{Combination sensitivity and lateral lemniscal nuclei}

An intriguing issue is that a clear tonotopic organization, which characterizes almost all large subcortical auditory nuclei, is not as apparent in VNLL and INLL of most species (see discussion in Benson and Cant, 2008). Assessments of tonotopic organization are often based on retrograde transport studies after tracer deposits in the IC. Our results provide a possible explanation: if INLL or VNLL neurons project to multiple IC frequency representations, any tracer deposit will label NLL neurons in multiple frequency representations, obscuring a tonotopic order. This is exactly what has been observed in the mustached bat (Frisina et al., 1989; Wenstrup et al., 1994, 1999; this study). We conclude that VNLL and INLL are tonotopically organized, but that this organization 
A
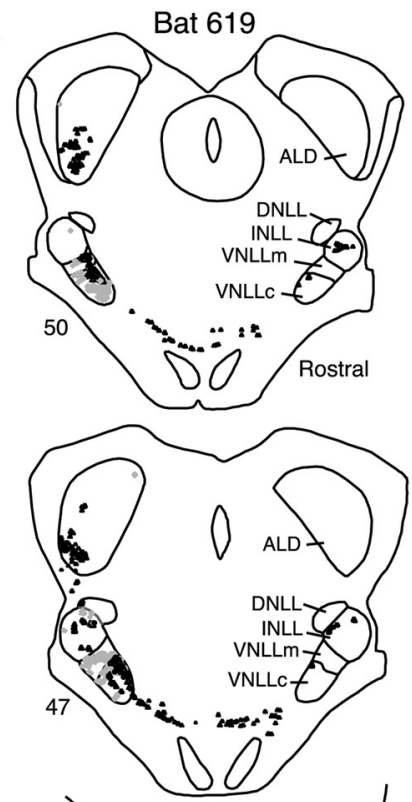

$\Delta \mathrm{FG}$ cell

$\triangle \mathrm{FR}$ bouton
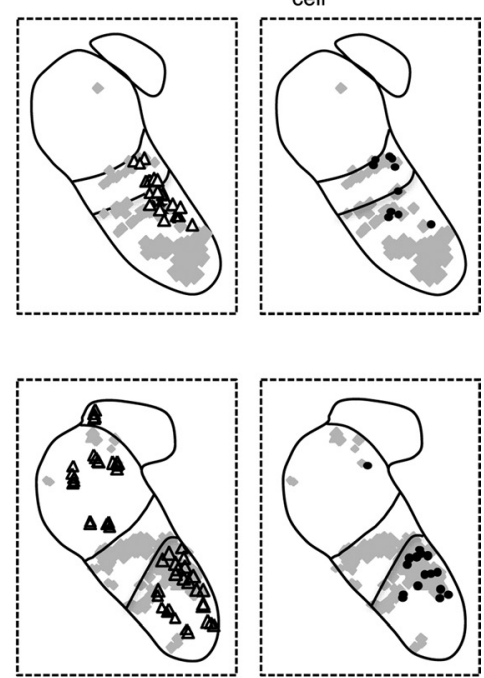

FG cell

- proximity labeled

cell

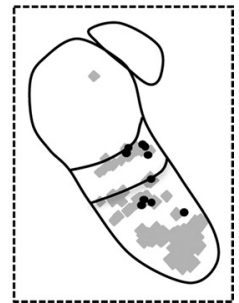

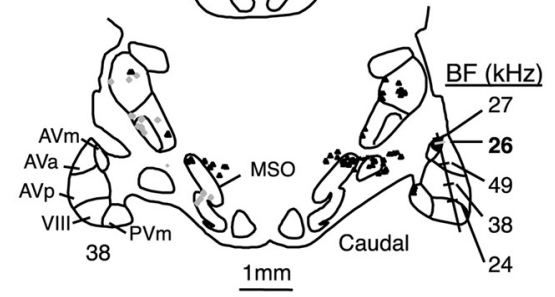

B
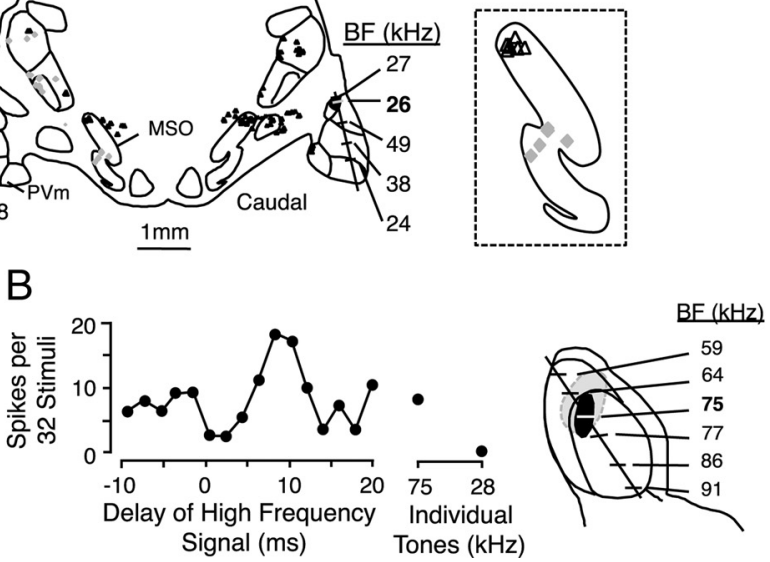

Tones $(\mathrm{kHz})$
Figure 10. A, Overlap of anterograde and retrograde labeling patterns in lateral lemniscal nuclei indicates low frequency input to facilitatory combination-sensitive sites in IC. A, Black open triangles show distribution of labeled boutons after the FR deposit in AVCN (illustrated in Fig. 8). Gray solid diamonds show distribution of retrograde (FG) labeling after two deposits at facilitated combination-sensitive recording sites in IC (see $\boldsymbol{B}$ ). Insets show higher-magnification views of labeling in NLL and MSO. Insets on the left, FR boutons and FG cells; insets on the right, proximity-labeled cells (black filled circles), defined as FG-labeled cells within $50 \mu \mathrm{m}$ of an FR bouton and indicating potential low-frequency AVCN input to neurons projecting to combination-sensitive IC neurons. Such overlap in labeling patterns occurs in VNLLC, VNLLm, and INLL, but not in MSO, LSO, or IC. ALD, Anterolateral division of the central nucleus of the inferior colliculus, representing frequencies below $\sim 57 \mathrm{kHz}$; AVa, anterior division of anteroventral cochlear nucleus; AVm, marginal division of anteroventral cochlear nucleus; AVp, posterior division of anteroventral cochlear nucleus; PVm, medial division of posteroventral cochlear nucleus; VIII, root of eighth cranial nerve. $\boldsymbol{B}$, Delay sensitivity function reveals combination-sensitive facilitation at IC recording site. The IC in this case received a second deposit at another combinationsensitive recording site.

is obscured by projections to disparate IC frequency representations.

A different form of combination sensitivity leads to inhibition of the high-frequency response when a low-frequency signal is also present (again, within a specific temporal window). While this inhibitory combination sensitivity is observed in the IC, it arises in INLL and perhaps VNLL. Specifically, the excitatory response of high-BF INLL neurons is inhibited by a lowfrequency, glycinergic input from the MNTB (Peterson et al., 2009; Yavuzoglu et al., 2010). These INLL cells likely provide glutamatergic projections to the IC that confer similar combination sensitivity onto IC cells. Thus, INLL and the VNLL appear to play essential roles in circuits underlying the two forms of combination sensitivity that have been observed in the IC.
VNLL and INLL are prominent nuclei across mammals (Helfert and Aschoff, 1997; Oertel and Wickesberg, 2002), but the nuclei are often relatively larger and the subdivisions more readily distinguished in echolocating animals. While it is reasonable to assume that these features may be related to echolocation, this provides little insight into the role of these nuclei in nonecholocating animals. In both echolocating and nonecholocating mammals, VNLL and INLL provide a substantial part of the ascending auditory inputs to the IC (Brunso-Bechtold et al., 1981; Moore, 1988; Cant and Benson, 2006), including a majority of the glycinergic input (Saint Marie and Baker, 1990). In a review of the VNLL, Oertel and Wickesberg (2002) suggested that both the nature of the inputs and the response properties of VNLL cells are consistent with a role in pattern recognition, including analysis of the complex temporal intervals present in speech. We propose that VNLL and INLL play an essential role in establishing combination sensitivity across species. This includes both facilitative combination sensitivity, as examined here, and inhibitory combination sensitivity (Yavuzoglu et al., 2010). These forms of analysis are clearly important in echolocation, but we expect that both forms of combination sensitivity contribute to the analysis of complex sounds including, but not limited to, vocalizations.

\section{References}

Arthur RM, Pfeiffer RR, Suga N (1971) Properties of 'two-tone inhibition' in primary auditory neurones. J Physiol 212:593-609.

Benson CG, Cant NB (2008) The ventral nucleus of the lateral lemniscus of the gerbil ( $M e$ riones unguiculatus): organization of connections with the cochlear nucleus and the inferior colliculus. J Comp Neurol 510:673-690.

Boothroyd A, Mulhearn B, Gong J, Ostroff J (1996) Effects of spectral smearing on phoneme and word recognition. J Acoust Soc Am 100:1807-1818.

Brunso-Bechtold JK, Thompson GC, Masterton RB (1981) HRP study of the organization of auditory afferents ascending to central nucleus of inferior colliculus in cat. J Comp Neurol 197:705-722.

Cant NB, Benson CG (2006) Organization of the inferior colliculus of the gerbil (Meriones unguiculatus): differences in distribution of projections from the cochlear nuclei and the superior olivary complex. J Comp Neurol 495:511-528.

Covey E, Casseday JH (1991) The monaural nuclei of the lateral lemniscus in an echolocating bat: parallel pathways for analyzing temporal features of sound. J Neurosci 11:3456-3470.

Covey E, Vater M, Casseday JH (1991) Binaural properties of single units in the superior olivary complex of the mustached bat. J Neurophysiol 66:1080-1094.

Esser KH, Condon CJ, Suga N, Kanwal JS (1997) Syntax processing by auditory cortical neurons in the FM-FM area of the mustached bat Pteronotus parnellii. Proc Natl Acad Sci U S A 94:14019-14024.

Frisina RD, O’Neill WE, Zettel ML (1989) Functional organization of mus- 
tached bat inferior colliculus. II. Connections of the FM2 region. J Comp Neurol 284:85-107.

Fuzessery ZM, Feng AS (1983) Mating call selectivity in the thalamus and midbrain of the leopard frog (Rana p. pipiens): Single and multiunit analyses. J Comp Physiol 150:333-344

Gans D, Sheykholeslami K, Peterson DC, Wenstrup J (2009) Temporal features of spectral integration in the inferior colliculus: effects of stimulus duration and rise time. J Neurophysiol 102:167-180.

Genzel D, Wiegrebe L (2008) Time-variant spectral peak and notch detection in echolocation-call sequences in bats. J Exp Biol 211:9-14.

Grothe B, Vater M, Casseday JH, Covey E (1992) Monaural interaction of excitation and inhibition in the medial superior olive of the mustached bat: an adaptation for biosonar. Proc Natl Acad Sci U S A 89:5108-5112.

Hebrank J, Wright D (1974) Spectral cues used in the localization of sound sources on the median plane. J Acoust Soc Am 56:1829-1834.

Helfert RH, Aschoff A (1997) Superior olivary complex and nuclei of the lateral lemniscus. In: The central auditory system: (Ehret G, Romand R, eds), pp 193-258. New York: Oxford UP.

Kadia SC, Wang X (2003) Spectral integration in A1 of awake primates: neurons with singleand multipeaked tuning characteristics. J Neurophysiol 89:1603-1622.

Kemmer M, Vater M (1997) The distribution of GABA and glycine immunostaining in the cochlear nucleus of the mustached bat (Pteronotus parnellii). Cell Tissue Res 287:487-506.

Kiang NY, Moxon EC (1974) Tails of tuning curves of auditory-nerve fibers. J Acoust Soc Am 55:620-630.

Knudsen EI, Konishi M (1979) Mechanisms of sound localization in the barn owl (Tyto alba) J Comp Physiol 133:13-21.

Kössl M, Vater M (1990) Tonotopic organization of the cochlear nucleus of the mustache bat Pteronotus parnellii. J Comp Physiol A 166:695-709.

Margoliash D, Fortune ES (1992) Temporal and harmonic combination-sensitive neurons in the zebra finch's HVc. J Neurosci 12:4309-4326.

Marsh RA, Nataraj K, Gans D, Portfors CV, Wenstrup JJ (2006) Auditory responses in the cochlear nucleus of awake mustached bats: precursors to spectral integration in the auditory midbrain. J Neurophysiol 95:88-105.

Metherate R, Kaur S, Kawai H, Lazar R, Liang K, Rose HJ (2005) Spectral integration in auditory cortex: mechanisms and modulation. Hear Res 206:146-158.

Middlebrooks JC (1992) Narrow-band sound localization related to external ear acoustics. J Acoust Soc Am 92:2607-2624.

Mittmann DH, Wenstrup JJ (1995) Combination-sensitive neurons in the inferior colliculus. Hear Res 90:185-191.

Moore BC (2008) Basic auditory processes involved in the analysis of speech sounds. Philos Trans R Soc Lond B Biol Sci 363:947-963.

Moore DR (1988) Auditory brainstem of the ferret: sources of projections to the inferior colliculus. J Comp Neurol 269:342-354.

Nataraj K, Wenstrup JJ (2005) Roles of inhibition in creating complex auditory responses in the inferior colliculus: facilitated combinationsensitive neurons. J Neurophysiol 93:3294-3312.

Oertel D, Wickesberg RE (2002) Ascending pathways through ventral nuclei of the lateral lemniscus and their possible role in pattern recognition in natural sounds. In: Integrative functions in the mammalian auditory pathway, vol 15. (Oertel D, et al, eds), pp 207-237. New York: Springer.
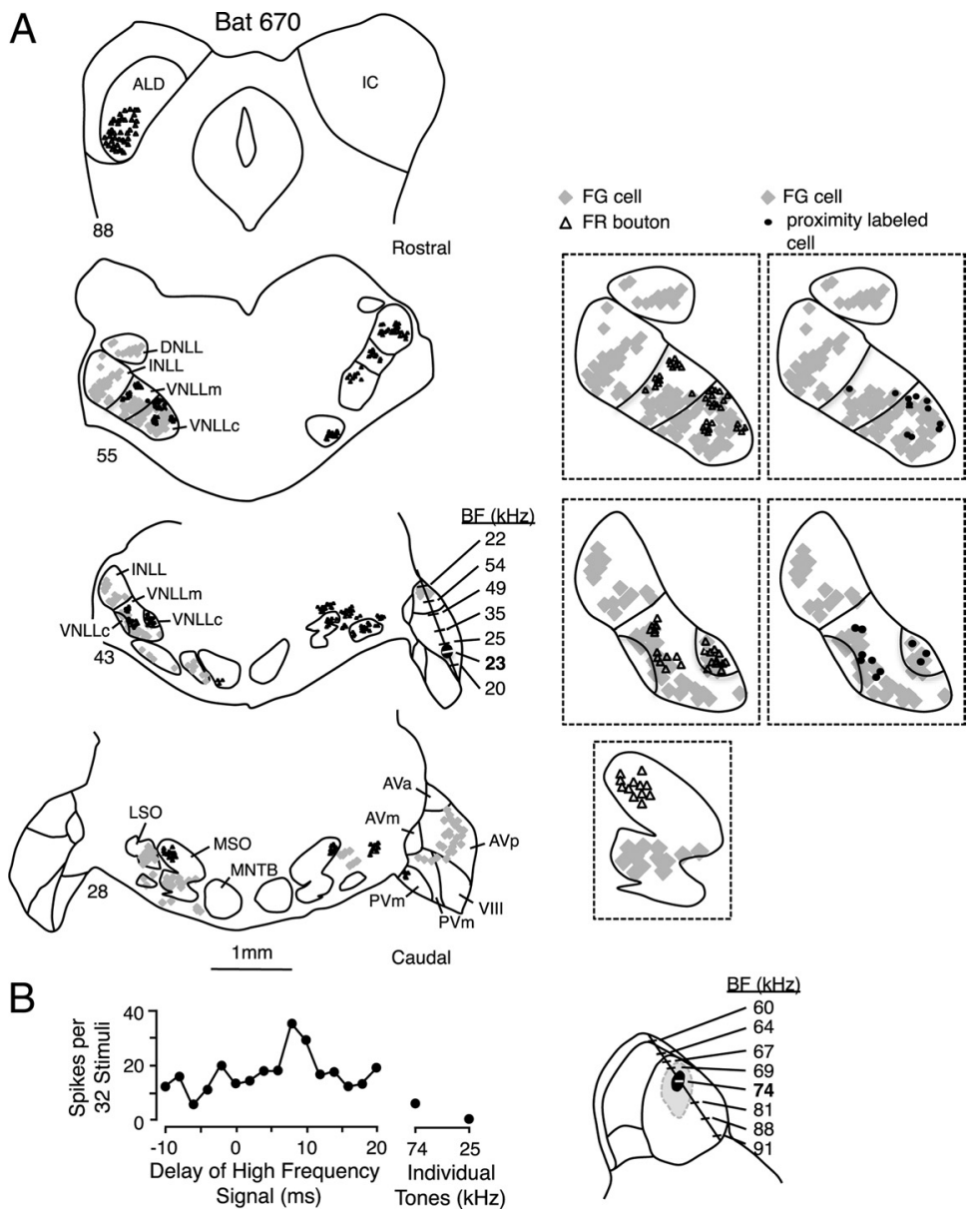

Figure 11. A, Overlap of anterograde and retrograde labeling patterns in lateral lemniscal nuclei indicates low-frequency input combination-sensitive sites in IC. Black open triangles show distribution of labeled boutons after the FR deposit in (F) labeling after two deposits at facilitated combination-sensitiv ting potential low-frequency AVCN input to neurons projecting to combination-sensitive IC neurons. Such overlap in labeling 列 of anteroventral cochlear nucleus; AVp, posterior division of anteroventral cochlear nucleus; PVm, medial division of posteroventral cochlear nucleus; VIII, root of eighth cranial nerve. $\boldsymbol{B}$, Delay sensitivity function reveals combination-sensitive facilitation at IC recording site. The IC in this case received a second deposit at another combination-sensitive recording site.

Ohlemiller KK, Kanwal JS, Suga N (1996) Facilitative responses to species specific calls in cortical FM-FM neurons of the mustached bat. Neuroreport 7:1749-1755.

Olsen JF, Suga N (1991) Combination-sensitive neurons in the medial geniculate body of the mustached bat: encoding of target range information. J Neurophysiol 65:1275-1296.

O'Neill WE, Suga N (1979) Target range-sensitive neurons in the auditory cortex of the mustached bat. Science 203:69-73.

Park TJ, Dooling RJ (1985) Perception of species-specific contact calls by budgerigars (Melopsittacus undulatus). J Comp Psychol 99: 391-402.

Peterson DC, Voytenko S, Gans D, Galazyuk A, Wenstrup J (2008) Intracellular recordings from combination-sensitive neurons in the inferior colliculus. J Neurophysiol 100:629-645.

Peterson DC, Nataraj K, Wenstrup J (2009) Glycinergic inhibition creates a form of auditory spectral integration in nuclei of the lateral lemniscus. J Neurophysiol 102:1004-1016.

Populin LC, Yin TC (1998) Behavioral studies of sound localization in the cat. J Neurosci 18:2147-2160.

Portfors CV, Wenstrup JJ (1999) Delay-tuned neurons in the inferior colliculus of the mustached bat: implications for analyses of target distance. J Neurophysiol 82:1326-1338. 


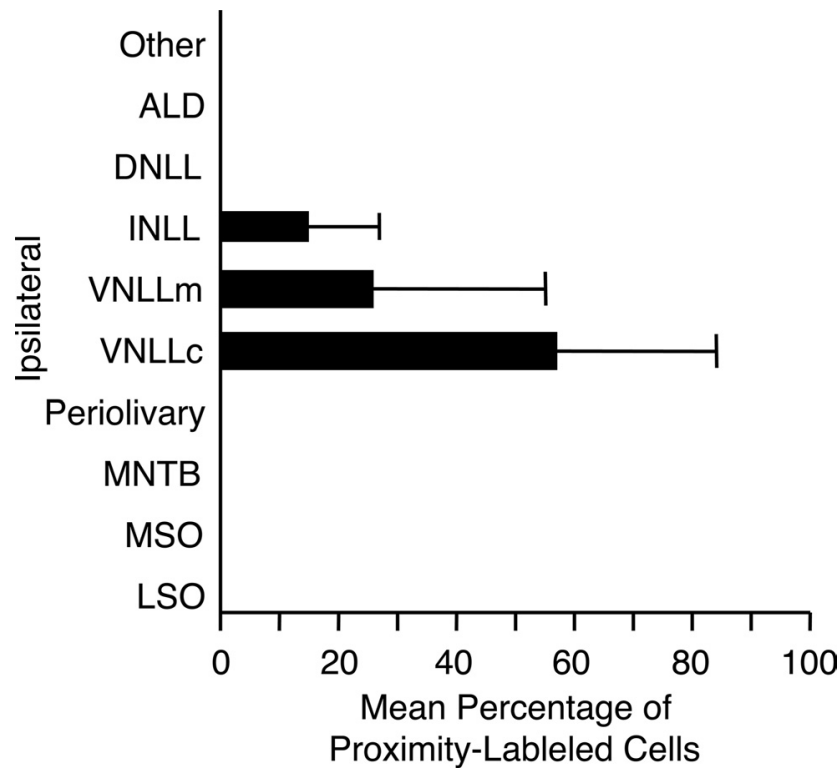

Figure 12. Only VNLL and INLL show proximity labeling that may underlie facilitatory combination-sensitive circuitry. The percentage of proximity-labeled cells in each nucleus was calculated by dividing the number of such cells in the nucleus by the total number in that animal; Mean Percentage of Proximity-Labeled Cells indicates the average percentage for that nucleus across five animals. Error bars indicate standard deviations. All listed auditory nuclei are ipsilateral to the IC deposit; no contralateral nucleus showed proximity labeling. ALD, Anterolateral division of the central nucleus of the inferior colliculus, representing frequencies below $\sim 57 \mathrm{kHz}$.

Portfors CV, Wenstrup JJ (2001) Responses to combinations of tones in the nuclei of lateral lemniscus. J Assoc Res Otolaryngol 2:104-117.

Portfors CV, Felix RA 2nd (2005) Spectral integration in the inferior colliculus of the CBA/CaJ mouse. Neuroscience 136:1159-1170.

Pow DV, Crook DK (1993) Extremely high titre polyclonal antisera against small neurotransmitter molecules: rapid production, characterisation and use in light- and electron-microscopic immunocytochemistry. J Neurosci Methods 48:51-63.

Pow DV, Wright LL, Vaney DI (1995) The immunocytochemical detection of amino-acid neurotransmitters in paraformaldehyde-fixed tissues. J Neurosci Methods 56: 115-123.

Ross LS, Pollak GD, Zook JM (1988) Origin of ascending projections to an isofrequency region of the mustache bat's inferior colliculus. J Comp Neurol 270:488-505.

Sachs MB, Kiang NY (1968) Two-tone inhibition in auditory-nerve fibers. J Acoust Soc Am 43:1120-1128.

Saint Marie RL, Baker RA (1990) Neurotransmitter-specific uptake and retrograde transport of $\left[{ }^{3} \mathrm{H}\right]$ glycine from the inferior colliculus by ipsilateral projections of the superior olivary complex and nuclei of the lateral lemniscus. Brain Res 524:244-253.

Sanchez JT, Gans D, Wenstrup JJ (2008) Glycinergic “inhibition” mediates selective excitatory response to combinations of sounds. J Neurosci 28:80-90.

Shannon RV, Fu QJ, Galvin J 3rd (2004) The number of spectral channels required for speech recognition depends on the difficulty of the listening situation. Acta Otolaryngol Suppl 552:50-54.

Simmons JA, Neretti N, Intrator N, Altes RA, Ferragamo MJ, Sanderson MI (2004) Delay accuracy in bat sonar is related to the reciprocal of normalized echo bandwidth, or Q. Proc Natl Acad Sci U S A 101:3638-3643.

Spirou GA, Young ED (1991) Organization of dorsal cochlear nucleus type IV unit response maps and their relationship to activation by bandlimited noise. J Neurophysiol 66:1750-1768.

Suga N (1989) Principles of auditory information-processing derived from neuroethology. J Exp Biol 146:277-286.

Suga N, O'Neill WE (1979) Neural axis representing target range in the auditory cortex of the mustache bat. Science 206:351-353.

Suga N, O'Neill WE, Manabe T (1978) Cortical neurons sensitive to combinations of information-bearing elements of biosonar signals in the mustache bat. Science 200:778-781.

Suga N, O’Neill WE, Kujirai K, Manabe T (1983) Specificity of combinationsensitive neurons for processing of complex biosonar signals in auditory cortex of the mustached bat. J Neurophysiol 49:1573-1626.

Takahashi T, Konishi M (1986) Selectivity for interaural time difference in the owl's midbrain. J Neurosci 6:3413-3422.

Vater M (1995) Ultrastructural and immunocytochemical observations on the superior olivary complex of the mustached bat. J Comp Neurol 358:155-180.

Vater M, Covey E, Casseday JH (1997) The columnar region of the ventral nucleus of the lateral lemniscus in the big brown bat (Eptesicus fuscus): synaptic arrangements and structural correlates of feedforward inhibitory function. Cell Tissue Res 289:223-233.

Wenstrup JJ (1999) Frequency organization and responses to complex sounds in the medial geniculate body of the mustached bat. J Neurophysiol 82:2528-2544.

Wenstrup JJ, Leroy SA (2001) Spectral integration in the inferior colliculus: role of glycinergic inhibition in response facilitation. J Neurosci 21:RC124.

Wenstrup JJ, Portfors CV (2011) Neural processing of target distance by echolocating bats: functional roles of the auditory midbrain. Neurosci Biobehav Rev. Advance online publication. Retrieved January 14, 2011. doi:10.1016/j.neubiorev.2010.12.015.

Wenstrup JJ, Larue DT, Winer JA (1994) Projections of physiologically defined subdivisions of the inferior colliculus in the mustached bat: targets in the medial geniculate body and extrathalamic nuclei. J Comp Neurol 346:207-236.

Wenstrup JJ, Mittmann DH, Grose CD (1999) Inputs to combinationsensitive neurons of the inferior colliculus. J Comp Neurol 409:509-528.

Winer JA, Larue DT, Pollak GD (1995) GABA and glycine in the central auditory system of the mustache bat: structural substrates for inhibitory neuronal organization. J Comp Neurol 355:317-353.

Yavuzoglu A, Schofield BR, Wenstrup JJ (2010) Substrates of auditory frequency integration in a nucleus of the lateral lemniscus. Neuroscience 169:906-919.

Young ED, Brownell WE (1976) Responses to tones and noise of single cells in dorsal cochlear nucleus of unanesthetized cats. J Neurophysiol 39:282-300.

Zook JM, Casseday JH (1982) Cytoarchitecture of auditory system in lower brainstem of the mustache bat, Pteronotus parnellii. J Comp Neurol 207:1-13.

Zook JM, Winer JA, Pollak GD, Bodenhamer RD (1985) Topology of the central nucleus of the mustache bat's inferior colliculus: Correlation of single unit response properties and neuronal architecture. J Comp Neurol 231:530-546. 